\title{
The effect of $\beta$-alanine and $\mathrm{NaHCO}_{3}$ co-ingestion on buffering capacity and exercise performance with high-intensity exercise in healthy males
}

\author{
Jessica Danaher · Tracey Gerber · R. Mark Wellard • \\ Christos G. Stathis
}

Received: 9 January 2014 / Accepted: 15 April 2014 / Published online: 16 May 2014

(C) The Author(s) 2014. This article is published with open access at Springerlink.com

\begin{abstract}
Introduction $\beta$-alanine $(\mathrm{BAl})$ and $\mathrm{NaHCO}_{3}(\mathrm{SB})$ ingestion may provide performance benefits by enhancing concentrations of their respective physiochemical buffer counterparts, muscle carnosine and blood bicarbonate, counteracting acidosis during intense exercise. This study examined the effect of BAl and SB co-supplementation as an ergogenic strategy during high-intensity exercise.

Methods Eight healthy males ingested either BAl (4.8 $\mathrm{g} \mathrm{day}^{-1}$ for 4 weeks, increased to $6.4 \mathrm{~g} \mathrm{day}^{-1}$ for 2 weeks) or placebo $(\mathrm{Pl})\left(\mathrm{CaCO}_{3}\right)$ for 6 weeks, in a crossover design (6-week washout between supplements). After each chronic supplementation period participants performed two trials, each consisting of two intense exercise tests performed over consecutive days. Trials were separated by 1 week and consisted of a repeated sprint ability (RSA) test and cycling capacity test at $110 \% \mathrm{Wmax}$ $\left(\mathrm{CCT}_{110} \%\right)$. Placebo (Pl) or SB (300 $\left.\mathrm{mg} \mathrm{kgbw}^{-1}\right)$ was ingested prior to exercise in a crossover design to creating four supplement conditions (BAl-Pl, BAl-SB, Pl-Pl, Pl-SB).
\end{abstract}

Communicated by Martin Flueck.

J. Danaher · T. Gerber · C. G. Stathis

College of Health and Biomedicine, Victoria University,

Melbourne, Australia

R. M. Wellard

Queensland University of Technology, Brisbane, Australia

C. G. Stathis $(\bowtie)$

Institute of Sport, Exercise and Active Living and the College

of Health and Biomedicine, Victoria University, Melbourne,

VIC 8001, Australia

e-mail: christos.stathis@vu.edu.au
Results Carnosine increased in the gastrocnemius $(n=5)$ $(p=0.03)$ and soleus $(n=5)(p=0.02)$ following $\mathrm{BAl}$ supplementation, and $\mathrm{Pl}-\mathrm{SB}$ and $\mathrm{BAl}-\mathrm{SB}$ ingestion elevated blood $\mathrm{HCO}_{3}{ }^{-}$concentrations $(p<0.01)$. Although buffering capacity was elevated following both BAl and SB ingestion, performance improvement was only observed with BAl-Pl and BAl-SB increasing time to exhaustion of the $\mathrm{CCT}_{110 \%}$ test 14 and $16 \%$, respectively, compared to $\mathrm{Pl}-\mathrm{Pl}(p<0.01)$.

Conclusion Supplementation of BAl and SB elevated buffering potential by increasing muscle carnosine and blood bicarbonate levels, respectively. BAl ingestion improved performance during the $\mathrm{CCT}_{110} \%$, with no aggregating effect of SB supplementation $(p>0.05)$. Performance was not different between treatments during the RSA test.

Keywords Beta-alanine $\cdot$ Carnosine $\cdot$ Bicarbonate . High-intensity exercise $\cdot$ Metabolism

$\begin{array}{ll}\text { Abbreviations } \\ \text { ANOVA } & \text { Analysis of variance } \\ \text { APO } & \text { Average power output } \\ \text { BAl-Pl } & \beta \text {-Alanine }+ \text { calcium carbonate } \\ \text { BAl-SB }^{-} & \beta \text {-Alanine }+ \text { sodium bicarbonate } \\ \text { CCT }_{110 \%} & \text { Cycling capacity test } \\ \text { HIE } & \text { High-intensity exercise } \\ \text { MRS } & \text { Magnetic resonance spectroscopy } \\ \text { Pl-P1 } & \text { Calcium carbonate }+ \text { calcium carbonate } \\ \text { Pl-SB } & \text { Calcium carbonate }+ \text { sodium bicarbonate } \\ \text { PPO } & \text { Peak power output } \\ \text { RSA } & \text { Repeated sprint ability } \\ \text { TTE } & \text { Time to exhaustion } \\ \text { VO }_{2 p e a k} & \text { Peak oxygen consumption }\end{array}$




\section{Introduction}

High-intensity exercise (HIE) results in the accumulation of glycolytic metabolites as a consequence of anaerobic metabolism during times of limited oxygen availability to the working cell (Coso et al. 2010; Sweeney et al. 2010). Although not the primary cause, an increased acidity of the working cells caused by hydrogen ions $\left(\mathrm{H}^{+}\right)$accumulation as by-products of anaerobic metabolism has potentially deleterious effects implicated in fatigue (Begum et al. 2005; Bishop et al. 2004) and can lead to significant impairments to exercise performance at high intensities (Robergs et al. 2005). Potential limiting factors resulting from acidosis include direct influences on contraction within the muscle cell due to competition of the $\mathrm{H}^{+}$with Calcium ion $\left(\mathrm{Ca}^{2+}\right)$ (Fabiato and Fabiato 1978), inhibition of glycolytic enzymes (Sutton et al. 1981) and PCr recovery (Harris et al. 1976), and its interference to the buffering process (Sahlin and Harris 2011).

Intramuscular $\mathrm{pH}$ homeostasis is achieved by active and passive transport of $\mathrm{H}^{+}$out of the muscle cell into the surrounding interstitium by physiochemical buffers, which work to maintain $\mathrm{pH}$ within a range for optimal enzyme function by tempering the effect of acidosis in the intracellular and extracellular milieu during HIE (Hill et al. 2007; Siegler et al. 2010). Physiochemical buffers include muscle carnosine, which plays an immediate role due to its intracellular location, and blood bicarbonate $\left(\mathrm{HCO}_{3}{ }^{-}\right)$, which has an extracellular presence (Sale et al. 2010).

Carnosine ( $\beta$-alanyl-L-histidine) is found in relatively high concentrations in human skeletal muscles at around 5-10 mmol kg ${ }^{-1}$ wet weight (ww) (Boldyrev and Severin 1990; Harris et al. 2006; Hill et al. 2007). It is endogenously synthesised from $\beta$-alanine (the limiting precursor in its synthesis) and L-histidine, and contributes between 8 and $15 \%$ of the endogenous $\mathrm{H}^{+}$buffering capacity (Van Thienen et al. 2009; Baguet et al. 2010). Its synthesis is slow and can take several weeks to accumulate in the muscle, warranting the need for chronic supplementation of $\beta$-alanine (Baguet et al. 2009; Derave et al. 2007). Increases in intense exercise performance following $\beta$-alanine ingestion indicate the potential of its influence on the intracellular $\mathrm{pH}$ buffer capacity via elevated carnosine concentrations (Hill et al. 2007; Sale et al. 2010; Sweeney et al. 2010). Although specific cellular mechanism(s) of the action and the consequential benefit to exercise performance remains unclear, elevated carnosine has also been linked to various additional cellular functions including, the regulation of $\mathrm{Ca}^{2+}$ sensitivity (Boldyrev and Severin 1990; Johnson and Aldstaft 1984), the protection of protein glycosylation by acting as a sacrificial peptide (Hipkiss et al. 1995), the inhibition of protein cross-linking by reacting with protein-carbonyl groups (Hipkiss 2000), and free radical scavenging by acting as an antioxidant (Chasovnikova et al. 1990).

Blood $\mathrm{HCO}_{3}{ }^{-}$is important for maintaining the homeostatic acid-base balance in the body (McNaughton et al. 2008). It also plays a role in attenuating the influence of the acute acid load following intense exercise once intracellular buffering capacity is exceeded and $\mathrm{H}^{+}$diffuses into the bloodstream (Juel et al. 2004; Swank and Robertson 1989), and may inevitably assist in postponing the potential effects of fatigue (McNaughton et al. 2008; Swank and Robertson 1989). Thus, the concentration of $\mathrm{HCO}_{3}^{-}$present in the blood prior to exercise potentially influences both extracellular buffering capacity and the extent to which the $\mathrm{H}^{+}$is able to diffuse out of the active muscle (Price et al. 2003). $\mathrm{NaHCO}_{3}$ ingestion has the potential to influence skeletal muscle function and prolong performance due to an improvement in blood buffering potential via the creation of an enhanced electro-chemical $\mathrm{pH}$ gradient between the intracellular and extracellular milieu, consequently allowing for a greater efflux of $\mathrm{H}^{+}$out of the muscle cell (Bishop et al. 2004; Price et al. 2003).

Combined nutritional strategies which enhance muscle carnosine and blood $\mathrm{HCO}_{3}^{-}$content, such as oral supplementation of $\beta$-alanine and $\mathrm{NaHCO}_{3}$, respectively, are therefore potentially ergogenic, particularly during HIE protocols with high glycolytic contribution, where acidosis may be a limiting factor to exercise performance (Price and Simons 2010; Sale et al. 2011). Although many previous studies have investigated the use of either $\beta$-alanine or $\mathrm{NaHCO}_{3}$ independently in improving parameters associated with HIE, little is known regarding the ergogenic effects of their co-ingestion.

Whilst recently published reports have examined the effects of this ergogenic strategy on continuous bout HIE (Bellinger et al. 2012; Sale et al. 2011), no study to date has, to the authors knowledge, investigated its effects on intermittent HIE or implemented a crossover design to account for individual variability. The aim of this study was therefore to investigate the effect of the co-consumption of $\beta$-alanine and $\mathrm{NaHCO}_{3}$ on HIE performance in healthy males. It was hypothesised that manipulating intra- and extracellular buffering capacity, via the coingestion of $\beta$-alanine and $\mathrm{NaHCO}_{3}$, respectively, will improve HIE performance variables compared to the placebo condition or the ingestion of either supplement alone. The co-consumption of $\beta$-alanine and $\mathrm{NaHCO}_{3}$ will buffer acidic by-products generated from anaerobic glycolysis, and thus enhance the ability to perform during both an intense intermittent and continuous exercise bout. 


\section{Methods}

Participants

Eight apparently healthy, recreationally active males $\left(26.2 \pm 1.9\right.$ year; $79.8 \pm 2.11 \mathrm{~kg} ; 179.0 \pm 2.2 \mathrm{~cm} ; \mathrm{VO}_{2 \text { peak }}$ $51.0 \pm 2.5 \mathrm{ml} \mathrm{kg}^{-1} \mathrm{~min}^{-1}$ ) volunteered to take part in this study, which was approved by the Victoria University Human Research Ethics Committee (HRETH 11/11) and performed in accordance with the ethical standards set out in the 1964 Declaration of Helsinki. After completing a medical questionnaire, each participant signed informed consent forms and presented for preliminary testing.

Participants were asked to refrain from consuming caffeine and alcohol, and from undertaking strenuous exercise $24 \mathrm{~h}$ prior to all experimental trials. Participants recorded their dietary intake $24 \mathrm{~h}$ before the first day of the first experimental trial, and were asked to consume a similar dietary intake the day prior to all subsequent trials. Experimental trials were conducted in the morning, approximately 10-12 $\mathrm{h}$ after the last meal. A standardised warm-up period of 5 min of cycling at $80 \mathrm{~W}$ was performed on an Excalibur Lode Cycle ergometer (Netherlands) before each trial, followed by $5 \mathrm{~min}$ rest. All tests were followed by $60 \mathrm{~min}$ passive recovery in a supine position.

\section{Preliminary testing}

Peak oxygen consumption $\left(\mathrm{VO}_{2 \text { peak }}\right)$ was determined approximately 1 week before the beginning of each chronic supplementation period. A standard graded exercise protocol on a Excalibur Lode Cycle ergometer (Netherlands) of $3 \times 3$ min sub-maximal workloads at 50, 100 and $150 \mathrm{~W}$ followed by successive 1-min workload increments of $25 \mathrm{~W}$ until volitional exhaustion. Participants were encouraged to maintain a pedal frequency between 80 and 100 RPM and the test was terminated when RPM could not be maintained $>80$ RPM for a period of $5 \mathrm{~s}$. Expired air was directed by a Hans Rudolph valve via a ventilometer (Moxus; AEI Technologies, Pennsylvania, USA) into a mixing chamber and analysed for oxygen and carbon dioxide content. Prior to each $\mathrm{VO}_{2 \text { peak }}$ test, the gas analyser was calibrated using commercially prepared gas mixtures (BOC Gases, Australia). Following preliminary testing, participants were familiarised with the exercise protocols in which they would be participating in during the experimental trials.

In vivo magnetic resonance spectroscopy (MRS) analysis

MRS was used in this study as a non-invasive quantification of human muscle carnosine before and after the $\beta$-alanine and placebo chronic supplementation periods in $n=5$ participants (due to access and availability of MRS). These measurements were made on a whole body proton 3 tesla scanner (Siemens Trio Tim, Germany) equipped with a flexible knee coil at The Royal Children's Hospital (Melbourne, Australia). Muscle carnosine concentration was quantified in the gastrocnemius and soleus muscles of the right leg throughout the study using a voxel of dimension $40 \mathrm{~mm} \times 30 \mathrm{~mm} \times 12 \mathrm{~mm}$. Data were acquired using a standard PRESS sequence with an echo time of $30 \mathrm{~ms}$, a relaxation delay of 2,000 ms and 176 transients. Muscle carnosine concentration was determined by comparing data recorded from the gastrocnemius and soleus muscles to a standard curve generated from external carnosine reference phantoms for absolute quantification. External carnosine reference phantoms contained $6.32,30.00$ and $58.93 \mathrm{mM}$ carnosine (Sigma) with sodium azide $\left(\mathrm{NaN}_{3}\right)$ as an antibacterial agent. No correction was made for differences in relaxation time.

A line broadening of $1 \mathrm{~Hz}$ was applied to the data prior to Fourier transformation to reduce any noise which may have altered the accuracy of results. Manual phasing was then applied before line fitting the spectra of each scan with an AMARES algorithm (jMRUI, version 4.0, build 113) (Naressi et al. 2001; Vanhamme et al. 1997). Peak amplitude (arbitrary scale) and linewidth (lw) results attained through this algorithm were then used to determine the signal intensity (area of each peak) using a Lorentzian equation; (I, Intensity; w, $0.5 \times \mathrm{lw}$ at $1 / 2$ maximum height; $2 \theta_{0}$, position of peak max):

$I(2 \theta)=w^{2} / w^{2}+\left(2 \theta-2 \theta_{0}\right)^{2}$.

Each spectrum contained two peaks, corresponding to the $\mathrm{C}_{2}-\mathrm{H}$ and $\mathrm{C}_{4}-\mathrm{H}$ imidazole protons of carnosine, and therefore the signal intensity of each peak was combined after adjusting to account for the number of protons in the structures giving rise to the peaks. A standard curve was then generated based on the concentration of each phantom scan and the signal intensity (area calculated from the phantom peak). The apparent concentration of carnosine from the gastrocnemius and soleus muscle scans was then determined with reference to the standard curve.

\section{Supplementation protocols}

The dual supplementation, double-blind, crossover experimental design was randomised for both $\beta$-alanine vs. placebo and $\mathrm{NaHCO}_{3}$ vs. placebo (Fig. 1) and all participants received the four supplement combinations. Specifically, there were two periods of 6-week chronic supplementation of capsulated $\beta$-alanine (Musashi, Australia) or the placebo calcium carbonate $\left(\mathrm{CaCO}_{3}\right)(\mathrm{E} 170$, Melbourne Food Ingredient Depot, Australia) which were separated by a minimum washout period of 6 weeks (no supplementation). 


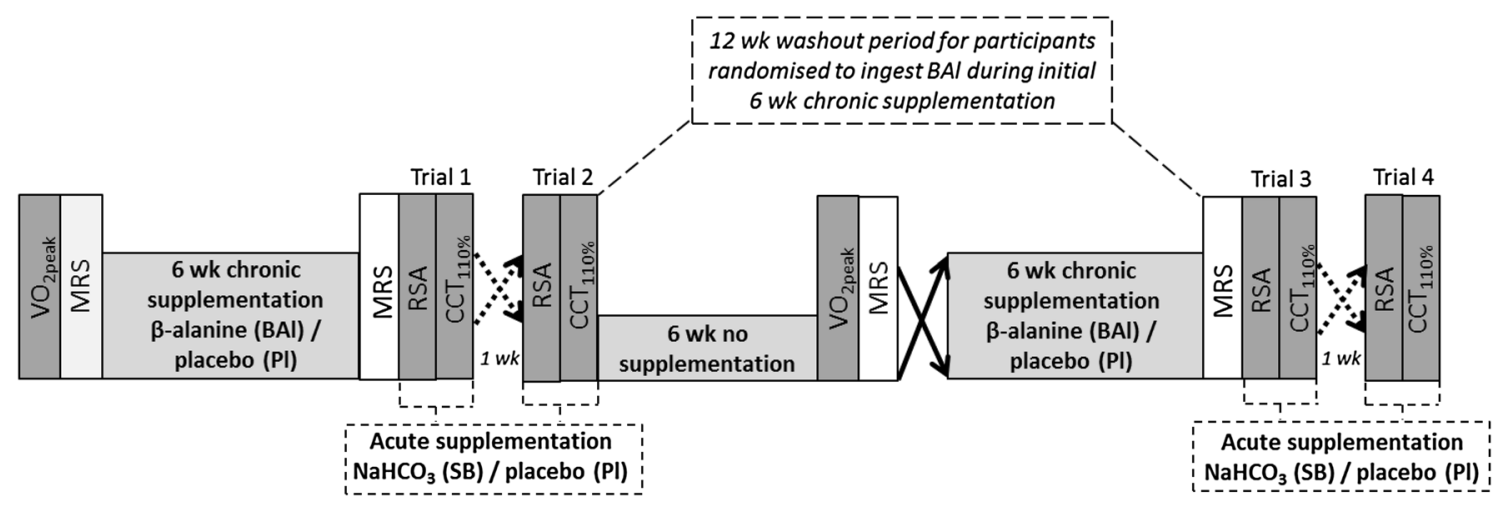

Fig. 1 Design of the study. Each trial consisted of two exercise tests performed over consecutive days. A total of 12 weeks between trials 2 and 3 was implemented to ensure adequate supplement washout time participants randomised to ingest $\beta$-alanine during the initial chronic supplementation. MRS Magnetic resonance spectroscopy,

The $\beta$-alanine supplementation procedure consisted of $4.8 \mathrm{~g} \mathrm{day}^{-1}(6 \times 800 \mathrm{mg})$ for 4 weeks and then $6.4 \mathrm{~g} \mathrm{day}^{-1}$ $(8 \times 800 \mathrm{mg})$ for 2 weeks. $\mathrm{CaCO}_{3}$ was administered in equal mass amounts as $\beta$-alanine. Participants were asked to spread their consumption of daily doses of $\beta$-alanine/ $\mathrm{CaCO}_{3}$ evenly throughout the day to avoid any possible paraesthesia. To facilitate compliance, a supplement diary was provided to participants upon commencing each chronic supplementation bout.

To investigate the superimposition of $\mathrm{NaHCO}_{3}$ with $\beta$-alanine, the acute administration of $\mathrm{NaHCO}_{3}$ (Sodibic, Aspen Pharmacare, Australia) occurred following each of the 6-week periods of $\beta$-alanine and placebo supplementation. This required two trials of either $300 \mathrm{mg} \mathrm{kgbw}^{-1}$ $\mathrm{NaHCO}_{3}$ or placebo $\left(\mathrm{CaCO}_{3}\right)$ supplementation in a randomised crossover design. This acute supplement ingestion was administered 90 min prior to the exercise bouts of the respective trials and was split into 6 equal doses over the first $50 \mathrm{~min}$ of the 90 -min pre-exercise period. Participants were asked to consume $300 \mathrm{ml}$ of water with each dose to assist in avoiding potential gastrointestinal side effects. These trials were separated by 7 days, performed in a randomised order, and $\beta$-alanine or respective placebo supplementation was extended until the second acute supplementation protocol was completed. Ultimately, the following four supplementation combinations were generated: $\beta$-alanine $+\mathrm{CaCO}_{3}(\mathrm{BAl}-\mathrm{Pl}), \beta$-alanine $+\mathrm{NaHCO}_{3}(\mathrm{BAl}-$ $\mathrm{SB}), \mathrm{CaCO}_{3}+\mathrm{CaCO}_{3}(\mathrm{Pl}-\mathrm{Pl})$, and $\mathrm{CaCO}_{3}+\mathrm{NaHCO}_{3}$ (Pl-SB).

\section{Experimental trial protocols}

Participants were asked to complete 2 exercise tests, over consecutive days, at the end of each of the aforementioned four co-supplement periods (Fig. 1). These tests were
RSA repeated sprint ability test, $C C T_{110 \%}$ cycling capacity test. Solid arrows depict crossover between acute supplementation (Pl and SB). Dotted arrows depict crossover between chronic supplementation (BAl and $\mathrm{Pl})$

designed to produce high acid loads whilst allowing examining the effect of supplementation on both intermittent and continuous HIE. The first test, a repeated sprint ability test (RSA), consisted of 5 repeats of $6 \mathrm{~s}$ maximal effort cycling bouts separated by $24 \mathrm{~s}$ rest (1:4 work-to-rest ratio). Peak and average power output were recorded after each sprint. This test was performed using a Wattbike Pro (British Cycling, Nottingham) and the same level of resistance was set for all trials (Air Brake 7.0). All sprints were performed in the standing position beginning with level pedals and the right leg forward, and with strong verbal encouragement. During the $24 \mathrm{~s}$ recovery intervals between sprints, participants were instructed to rest passively.

The second exercise test was a cycling capacity test $\left(\mathrm{CCT}_{110} \%\right)$ performed at $110 \%$ of the workload achieved at $\mathrm{VO}_{2 \text { peak }}$ prior to each chronic supplementation period. Participants were required to cycle in a seated position at $110 \%$ of their peak power output $\left(W_{\max }\right)(335.9 \pm 16.6 \mathrm{~W})$ between 80 and $100 \mathrm{rpm}$ for as long as possible. The clock was stopped at the point where their cadence could not be sustained above $80 \mathrm{rpm}$ (time to exhaustion; TTE). To overcome the inertia of the electromagnetic resistance of initial exercise at low cadence, the protocol on the electrically loaded bike the beginning of the $\mathrm{CCT}_{110} \%$ was modified to begin with $15 \mathrm{~s}$ of $80 \% W_{\max }$ and $15 \mathrm{~s}$ of $90 \% W_{\max }$ before $110 \% W_{\max }$ was attained.

Blood sampling, treatment and analysis

Blood was sampled from a vein in the antecubital space and the cannula was kept patent with isotonic saline $(0.9 \% \mathrm{NaCl}$, Pfizer). Blood was sampled at rest (pre- and post-NaHCO $\mathrm{N}_{3}$ /placebo supplementation), immediately after exercise and during a $60 \mathrm{~min}$ post exercise recovery period. One portion of the blood was immediately placed 
Fig. 2 Changes in carnosine concentration in the gastrocnemius (a) and soleus (b) pre- and post-6-week chronic supplementation of $\beta$-alanine (BAl) and placebo (Pl) for $n=5$. Values expressed as mean \pm SEM. $* p<0.05$ from $\mathrm{Pl}$ at 6 weeks (post), ${ }^{\#} p<0.05$ from pre-supplementation

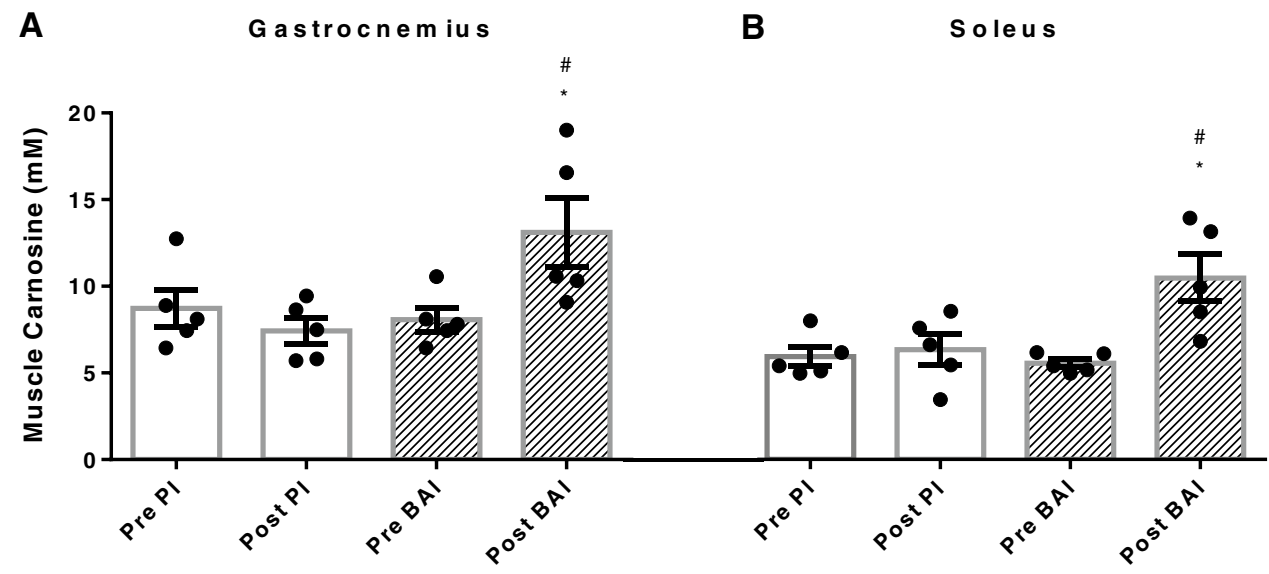

into lithium heparin (BD Vacutainer) tubes and centrifuged at 12,000 rpm for $2 \mathrm{~min}$. Plasma was decanted and stored at $-80{ }^{\circ} \mathrm{C}$ for later analysis of lactate concentration (YSI 2300 STAT; Yellow Springs Instruments, Ohio, USA). Blood $\mathrm{pH}$ and $\mathrm{HCO}_{3}{ }^{-}$were analysed immediately using a Radiometer ABL800 FLEX Blood Gas Analyser (ABL800 FLEX; Radiometer Medical, Brønshøj, Denmark).

\section{Statistical analysis}

Results are expressed as mean \pm standard error of the mean (SEM). Two-way analysis of variance (ANOVA) with repeated measures was performed (GraphPad Prism 6, Windows XP) one within group factor (time or sprint) and one between group factor (treatment), for blood, plasma and RSA data. Where there was an interaction between the factors, Tukey's post hoc analysis was performed. Oneway AVOVA with repeated measures was performed for $\mathrm{CCT}_{110} \%$ and muscle carnosine data (individual $\times$ treatment), with paired two-tailed $t$ tests when interactions between factors were found. The level of probability required to reject the null hypothesis was set at $p<0.05$.

\section{Results}

Muscle carnosine content

The carnosine concentration was elevated in the gastrocnemius and soleus muscles following 6 weeks of supplementation of $\beta$-alanine (Fig. 2). In the gastrocnemius muscle (Fig. 2a), the absolute carnosine was $8.08 \pm 0.68$ and $8.73 \pm 1.08 \mathrm{mM}$ in the $\beta$-alanine and placebo groups, before supplementation, respectively, and increased by $5.03 \pm 1.44 \mathrm{mM}(+62 \%$, to $13.11 \pm 1.97 \mathrm{mM}$; $p=0.03$ ) after supplementation, whereas it remained stable at $7.42 \pm 0.74 \mathrm{mM}(p=0.20)$ in the placebo group. Six weeks of chronic supplementation also caused the carnosine content of the soleus muscle (Fig. 2b) to increase by $4.92 \pm 1.28 \mathrm{mM}(+88 \%, 5.57 \pm 0.25$ to $10.48 \pm 1.35 \mathrm{mM} ; p=0.02)$ before and after supplementation, respectively, in the $\beta$-alanine group whilst the carnosine concentration of the placebo group did not change $(+7 \%, 5.94 \pm 0.56$ to $6.33 \pm 0.89 \mathrm{mM} ; p=0.6)$. Carnosine concentrations in both muscle groups were also significantly elevated compared to that of the placebo (Pl) condition following 6 weeks of supplementation [gastrocnemius, $+5.69 \pm 2.08(p=0.05)$; soleus $+4.15 \pm 0.78$ $(p=0.006)]$.

The washout protocol of carnosine from the muscle for participants who received $\beta$-alanine during the first chronic supplementation period $(n=3)$ was modified at the end of the experimental procedures, once the supplementation order randomisation was de-coded. This was done to ensure a minimum 12-week washout period upon cessation of $\beta$-alanine supplementation, as estimated from previous research (Baguet et al. 2009; Derave et al. 2007). In these participants, the post-supplementation sample in the second of the 6-week supplementation periods (placebo) was unmodified; however, values observed via MRS pre- $\beta$-alanine supplementation (initial MRS) were used for baseline data in these participants when examining the effect of chronic placebo supplementation. This was due to the second chronic supplementation period beginning during the 6 week of the washout period, and hence data collected here did not accurately reflected basal muscle carnosine concentrations. After a washout of 6 weeks, carnosine concentrations had decreased to $10.03 \pm 1.34 \mathrm{mM}(-19 \%)$ in the gastrocnemius and $6.54 \pm 0.60 \mathrm{mM}(-23 \%)$ in the soleus, at a rate of $-0.3 \mathrm{mM}$ week $^{-1}$ and $-0.4 \mathrm{mM}$ week $^{-1}$, respectively. Twelve weeks following cessation of $\beta$-alanine supplementation (end of second chronic supplementation period), muscle carnosine concentrations in both the gastrocnemius and soleus had returned to baseline concentrations, with the average rate of carnosine consistently decreasing in the 
Fig. 3 Blood $\mathrm{HCO}_{3}{ }^{-}$ $\left(\mathrm{mmol} \mathrm{L}^{-1}\right.$ ) changes for study groups during the repeated sprint ability (RSA) and cycling capacity test $\left(\mathrm{CCT}_{110 \%}\right)$. Values expressed as mean \pm SEM. $* p<0.05$ BAl-SB and Pl-SB from Pl-Pl, ${ }^{\#} p<0.05$ BAl$\mathrm{SB}$ and Pl-SB from PreEx0, ${ }^{+} p<0.05$ Pl-SB from PreEx0, thick vertical bar denotes exercise

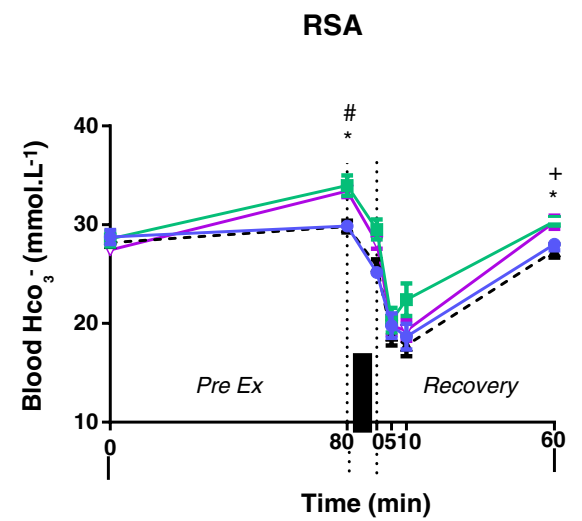

gastrocnemius by $0.5 \pm 0.03 \mathrm{mM}$ week ${ }^{-1}$ and in the soleus by $0.4 \pm 0.02 \mathrm{mM}^{-1}$ week $^{-1}$. This information demonstrates that a washout period of 12 weeks was adequate to employ a randomised crossover experimental design, with an overall average rate of carnosine concentration decrease following the cessation of $\beta$-alanine supplementation.

\section{Blood $\mathrm{HCO}_{3}{ }^{-}$concentration and $\mathrm{pH}$}

There were significant increases in blood $\mathrm{HCO}_{3}{ }^{-}$between baseline and the post-supplementation period in both trials involving $\mathrm{NaHCO}_{3}$ supplementation (Fig. 3, BAl-SB and Pl-SB) (average increase of $3.67 \pm 0.46 \mathrm{mmol} \mathrm{L}^{-1}$; $p \leq 0.0001)$. The placebo group showed no changes. The blood $\mathrm{HCO}_{3}{ }^{-}$in these groups was also significantly higher than that of the placebo condition (Pl-Pl) throughout both exercise bouts $(p \leq 0.01)$. No change was observed in blood $\mathrm{pH}$ between supplement groups or compared to baseline values prior to either exercise (Fig. 4). There was an increase in both blood $\mathrm{HCO}_{3}{ }^{-}$concentration and blood $\mathrm{pH}$ at the end of the recovery period following BAl-SB and Pl-SB compared to baseline values and the $\mathrm{Pl}-\mathrm{Pl}$ condition in both exercise bouts $(p \leq 0.01)$, with the exception of BAl-SB blood $\mathrm{HCO}_{3}{ }^{-}$between baseline and 60 min following RSA $(p=0.07))$. BAl-SB was the only treatment group to show significantly higher blood $\mathrm{pH}$ values (up to $0.07 \pm 0.01 \mathrm{pH}$ units; $p \leq 0.0001$ ) throughout the first $10 \mathrm{~min}$ of recovery compared to $\mathrm{Pl}-\mathrm{Pl}$, in both exercise tests.

\section{Repeated sprint ability test (RSA)}

No differences in peak power output (PPO) or average power output (APO) were observed between supplement groups during each of the 56-s sprints of the RSA test (Table 1). There were also no differences in the total work done between either or combined supplement groups with a comparison of total work done (TW) (BAl-Pl, 22,136.25 \pm 1,757.01 J; BAl$\mathrm{SB}, 22,487.00 \pm 1,724.30 \mathrm{~J}$; Pl-Pl, 23,586.00 $\pm 1,831.58 \mathrm{~J}$ Pl-SB, 23,068.50 $\pm 1,749.20 \mathrm{~J})$.
Cycling capacity test $\left(\mathrm{CCT}_{110 \%}\right)$

A greater TTE by $18.13 \pm 6.75 \mathrm{~s}(+14 \%)(p=0.005)$ was measured in the BAl-Pl compared to Pl-Pl (147 \pm 13.16 and $129 \pm 10.94$, respectively) during the $\mathrm{CCT}_{110} \%$ (Fig. 5). Although the addition of $\mathrm{NaHCO}_{3}$ to $\beta$-alanine further increased TTE by $2.75 \pm 4.61 \mathrm{~s}(+2 \%)(p=0.02$ compared to $\mathrm{Pl}-\mathrm{Pl})$, there was no statistical difference between BAl-Pl and BAl-SB $(p=0.57)$. No change in TTE was determined in Pl-SB treated group (132 $\pm 13.48 \mathrm{~s})$ $(+2 \%)$ compared to the $\mathrm{Pl}-\mathrm{Pl}$ condition $(p=0.51)$.

\section{Plasma lactate}

Plasma lactate concentrations $\left(\mathrm{mmol} \mathrm{L}^{-1}\right)$ were elevated in all treatment groups following the completion of exercise in both the RSA (data not shown) and $\mathrm{CCT}_{110 \%}$ (Fig. 6), and remained significantly elevated compared to baseline at the end of the recovery period. A difference was observed at the 5 th min of the recovery period following the $\mathrm{CCT}_{110} \%$ protocol, with BAl-SB higher than Pl-Pl by $2.66 \pm 1.20 \mathrm{mmol} \mathrm{L}^{-1}(p=0.04)$. Although the Pl-SB treated groups also showed an apparent increase in plasma lactate $\left(+2.28 \pm 1.61 \mathrm{mmol} \mathrm{L}^{-1}\right)$ following this test, this was non-significant $(p=0.18)$.

\section{Discussion}

This study demonstrated an increase in both intracellular and extracellular buffering potential following chronic $\beta$-alanine (6 weeks) and acute $\mathrm{NaHCO}_{3}$ (90 min) supplementation, respectively. Furthermore, the combined supplementation induced a similar buffering potential to that of the sum of the individual supplementation protocols. Improvements in exercise performance following the combined supplementation protocol, however, were equivocal and indicate that aggregating the supplementation protocol may not have a cumulative effect on physiochemical 
Table 1 Peak power output (PPO) and average power output (APO) (W) achieved for each of the $5 \times 6 \mathrm{~s}$ sprints between supplement groups during the repeated sprint ability (RSA) test

Values expressed as mean \pm SEM

\begin{tabular}{|c|c|c|c|c|}
\hline & BAl-Pl & BAl-SB & $\mathrm{Pl}-\mathrm{Pl}$ & Pl-SB \\
\hline \multicolumn{5}{|l|}{ PPO (W) } \\
\hline Sprint 1 & $987 \pm 74.70$ & $970 \pm 71.32$ & $1,028 \pm 70.53$ & $1,000 \pm 72.20$ \\
\hline Sprint 2 & $969 \pm 75.43$ & $976 \pm 84.57$ & $989 \pm 73.16$ & $1,018 \pm 70.28$ \\
\hline Sprint 3 & $948 \pm 72.27$ & $946 \pm 78.04$ & $966 \pm 61.07$ & $969 \pm 58.40$ \\
\hline Sprint 4 & $933 \pm 72.72$ & $927 \pm 69.18$ & $938 \pm 58.96$ & $911 \pm 58.60$ \\
\hline Sprint 5 & $884 \pm 73.99$ & $884 \pm 60.74$ & $913 \pm 51.96$ & $904 \pm 64.19$ \\
\hline \multicolumn{5}{|l|}{$\mathrm{APO}(\mathrm{W})$} \\
\hline Sprint 1 & $803 \pm 67.13$ & $813 \pm 64.23$ & $873 \pm 73.35$ & $857 \pm 64.13$ \\
\hline Sprint 2 & $774 \pm 61.74$ & $792 \pm 56.67$ & $826 \pm 69.25$ & $826 \pm 60.50$ \\
\hline Sprint 3 & $732 \pm 59.13$ & $749 \pm 59.51$ & $773 \pm 62.57$ & $757 \pm 61.34$ \\
\hline Sprint 4 & $709 \pm 54.24$ & $705 \pm 57.31$ & $749 \pm 57.35$ & $721 \pm 56.57$ \\
\hline Sprint 5 & $672 \pm 55.40$ & $690 \pm 53.18$ & $710 \pm 46.26$ & $684 \pm 56.45$ \\
\hline
\end{tabular}

buffering capacity. The improvement in the $\mathrm{CCT}_{110} \%$ performance and not the RSA protocol may be a reflection of the modality of HIE which may influence metabolism differently and consequently have different impacts on intracellular and extracellular buffering systems. Furthermore, it could demonstrate that fatigue may not be $\mathrm{pH}$ related in some instances of HIE.

Muscle carnosine concentrations increased by 62 and $88 \%$ in the gastrocnemius and soleus, respectively, following 6 weeks of supplementation with $\beta$-alanine. This is comparable to direct measures in vastus lateralis biopsies of untrained subjects (Harris et al. 2006; Hill et al. 2007) and indirect MRS gastrocnemius and soleus muscle of both trained (Derave et al. 2007) and untrained males (Baguet et al. 2009), following chronic supplementation of $\beta$-alanine. The increase in carnosine content in the current study was higher than previous reports using MRS technology, with Baguet et al. (2009) showing a 23 and $39 \%$ increase, and Derave et al. (2007) a 37 and $47 \%$ increase in the gastrocnemius and soleus muscles, respectively. This may be due to differences between $\beta$-alanine supplementation protocols where 6 weeks and a total dosage of $224 \mathrm{mg}$ were utilised in this study compared to shorter duration and lower dosages implemented in previous studies.

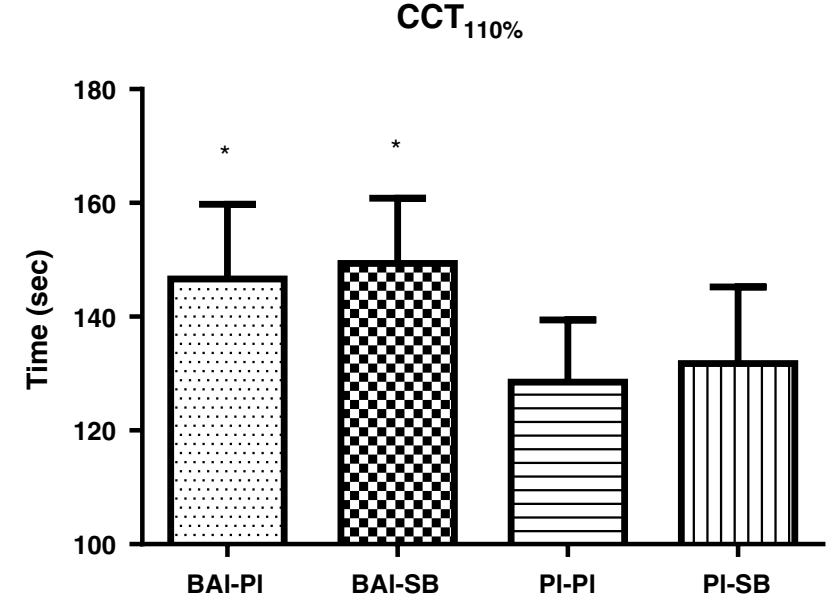

Fig. 5 Time to exhaustion (TTE) (s) results for the different supplement groups during the cycling capacity test $\left(\mathrm{CCT}_{110 \%}\right)$. Values expressed as mean \pm SEM. $* p<0.05$ from $\mathrm{Pl}-\mathrm{Pl}$

The efficacy of the $\mathrm{NaHCO}_{3}$ supplementation employed in this study was demonstrated by a significant increase in blood $\mathrm{HCO}_{3}{ }^{-}$following the 90 -min pre-exercise supplementation period in both the BAl-SB and Pl-SB groups
Fig. 4 Blood pH recorded for the different groups during the repeated sprint ability (RSA) and cycling capacity test $\left(\mathrm{CCT}_{110 \%}\right)$. Values expressed as mean \pm SEM. $* p<0.05$ BAl-SB and Pl-SB from Pl-Pl, ${ }^{\#} p<0.05 \mathrm{BAl}-\mathrm{SB}$ and Pl-SB from PreEx $0,{ }^{+} p<0.05 \mathrm{BAl}-\mathrm{SB}$ from $\mathrm{Pl}-\mathrm{Pl}$, thick vertical bar denotes exercise
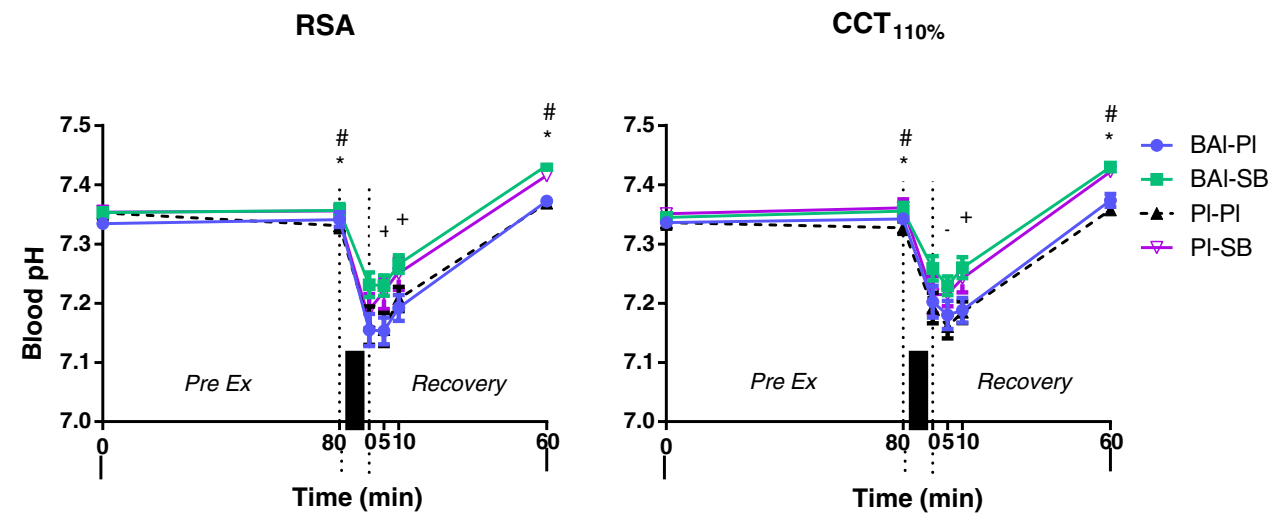


\section{$\mathrm{CCT}_{110 \%}$}

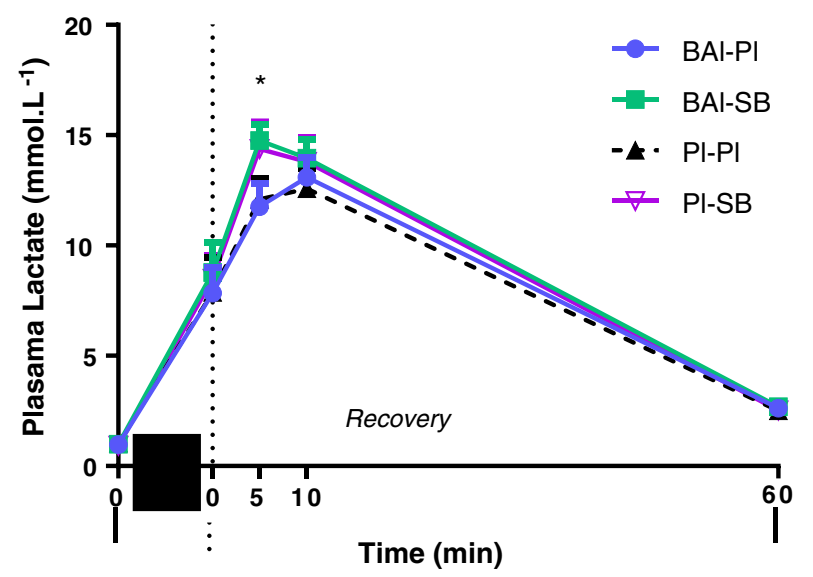

Fig. 6 Plasma lactate concentration $\left(\mathrm{mmol} \mathrm{L}^{-1}\right)$ for the different supplemental groups during the cycling capacity test $\left(\mathrm{CCT}_{110} \%\right)$. Values expressed as mean \pm SEM. $* p<0.05$ BAl-SB from Pl-Pl. Thick vertical bar denotes exercise

compared to placebo (Fig. 3). The elevation in blood $\mathrm{HCO}_{3}^{-}$in the current study $\left(5.99 \mathrm{mmol} \mathrm{L}{ }^{-1}\right)$ following $\mathrm{NaHCO}_{3}$ ingestion was of a similar magnitude to the mean increase of $5.3 \mathrm{mmol} \mathrm{L}^{-1}$ reported in a meta-analysis of human $\mathrm{NaHCO}_{3}$ loading studies which also used a $300 \mathrm{mg} \mathrm{kg}^{-1}$ dose (Matson and Vu Tran 1993). Although other investigations have reported plasma $\mathrm{pH}$ increases of $\sim 0.05$ units following similar $\mathrm{HCO}_{3}{ }^{-}$supplementation regimes, discrepancies in HIE performance have resulted in either positive (Bishop et al. 2004; Lindh et al. 2008; Siegler et al. 2010) or no change (Matsuura et al. 2007; Price et al. 2003; Sale et al. 2011). A lack of change in blood pH between pre-acute supplementation and pre-exercise was observed in this study, indicating that despite a significant rise in blood $\mathrm{HCO}_{3}^{-}$, metabolic alkalosis was not being achieved (see Fig. 4).

Ingestion of $\mathrm{NaHCO}_{3}$ elevated blood $\mathrm{pH}$ by $\sim 0.07 \mathrm{pH}$ units $60 \mathrm{~min}$ following exercise in both HIE tests, along with a tendency to cause higher blood $\mathrm{HCO}_{3}{ }^{-}$concentrations. This suggests that a longer time between acute supplementation and exercise commencing may have been more suitable in this study to induce an alkalotic state, which may in turn assist in improving HIE performance parameters. Extended ingestion protocols ( $>90 \mathrm{~min}$ ) have been previously investigated, with metabolic alkalinity achieved prior to exercise; however, the performance enhancing abilities of $\mathrm{NaHCO}_{3}$ even with longer supplementation protocols employed remain equivocal (Gaitonos et al. 1991; Sale et al. 2011).

To our knowledge, this is the first study to examine the co-ingestion of $\beta$-alanine and $\mathrm{NaHCO}_{3}$ on intermittent
HIE performance parameters. PPO and APO were not different between treatment groups during each of the $5 \times 6 \mathrm{~s}$ sprints performed during the RSA test, or in terms of total work performed. Gaitonos et al. (1991) also reported that $\mathrm{NaHCO}_{3}$ supplementation did not induce performance benefits when using a similar exercise protocol $(10 \times 6 \mathrm{~s}$ sprints every $36 \mathrm{~s}$ ) in male subjects. Although Gaitonos et al. (1991) assessed running performance, rather than cycling performance as used in this study; both used similar metabolic pathways in order for the effect of supplementation to be seen (Fitzsimons et al. 1993). This is contrary to an identical RSA exercise protocol employed by Bishop et al. (2004) demonstrating that the oral ingestion of $\mathrm{NaHCO}_{3}$ in active female hockey players increased PPO compared to the placebo condition during the last three of the $5 \times 6 \mathrm{~s}$ sprints performed, indicating a potential specific conditioning of training-dependant response.

Sweeney et al. (2010) examined the effect of 5 weeks $\beta$-alanine supplementation using a similar total dose (224 vs. $238 \mathrm{~g}$ ) on RSA in active males. Consistent with the present findings, $\beta$-alanine supplementation alone did not influence repeat sprint performance, with no change in power output observed. Co-ingestion with $\mathrm{NaHCO}_{3}$ in our study was unable to improve performance, demonstrating no influence of the treatment strategy on intermittent HIE.

The absence of a difference between treatments indicates either a potential gain in both intra- and extracellular buffing capacity, represented by observed elevations in muscle carnosine and blood bicarbonate, was either insufficient to bring about an increase in performance, or that decreases in $\mathrm{pH}$ is not the predominant limiting factor in intermittent HIE. Previous research observing high-intensity repeated sprint performance has also attributed fatigue to the availability and resynthesis of phosphocreatine (PCr), in addition to the effects of acidosis (Derave et al. 2010; Sweeney et al. 2010). During a single bout (6 s) of high-intensity exercise, the utilisation of $\mathrm{PCr}$ and anaerobic glycolysis each account for a majority of the energy source required (Sweeney et al. 2010). Repetition of short-duration sprints may result in a greater depletion of $\mathrm{PCr}$ than would occur during a single maximal effort (Derave et al. 2007). The rest between sprints during the RSA (24 s) may not have been long enough to resynthesis $\mathrm{PCr}$, as this process typically takes minutes rather than seconds (Derave et al. 2007). Hence, it is possible that $\mathrm{PCr}$ depletion may have been a greater cause of fatigue than acidosis during the RSA protocol implemented $(5 \times 6 \mathrm{~s}$ interspersed with $24 \mathrm{~s}$ rest), and as a result, any potential ergogenic effects of $\beta$-alanine and $\mathrm{NaHCO}_{3}$ as physiochemical buffers during intermittent HIE may not have been detectable.

$\beta$-alanine supplementation significantly increased TTE during the $\mathrm{CCT}_{110} \%$ test with a $14 \%$ improvement. This compares favourably with identical continuous HIE cycling 
protocols implemented by Sale et al. (2011) who also reported a $15 \%$ increase following a 4-week supplementation period, and Hill et al. (2007) who showed a $13 \%$ increased enhancement in TTE 4 weeks following supplementation, with a further $3 \%$ increase after a 6-week supplement extension. Cycling capacity protocols, such as the one employed in these studies, can be considered beneficial as they induce a large accumulation of acidic by-products resulting in a drop in intracellular $\mathrm{pH}$, such that the potentially ergogenic effects of $\beta$-alanine are influential.

In contrast to the effect of $\beta$-alanine on continuous HIE, $\mathrm{NaHCO}_{3}$ ingestion alone did not improve performance parameters relative to that of the Pl-Pl condition. Earlier studies show no effect of $\mathrm{NaHCO}_{3}$ on exercise performance using cycling protocols where participants cycle to exhaustion at both $110 \%$ (Robergs et al. 2005; Sale et al. 2011) and $120 \%$ $W_{\max }$ (Siegler et al. 2007) has previously been observed, despite the clear stimulus of increased $\mathrm{H}^{+}$production in the working muscle cells. Consistent with the report of Sale et al. (2011), the results of this investigation support the ability to increase continuous HIE capacity following the co-ingestion of $\beta$-alanine and $\mathrm{NaHCO}_{3}$ above that of $\mathrm{NaHCO}_{3}$ alone, but not compared to $\beta$-alanine alone, with only a $2 \%$ increase $(\sim 3 \mathrm{~s})$ in TTE attained. Whilst this magnitude of elevation was not statistically significant (with $95 \%$ confidence limits), it could be postulated that in terms of athletic performance small increases in TTE may still be important.

An increased buffering capacity may improve metabolic conditions for sustained elevated glycolytic energy production during the final stages of maximal effort exercise, and result in elevation of lactate ions in the active muscles and blood (Price and Simons 2010; Siegler et al. 2010; Van Thienen et al. 2009). Immediately following both the RSA test and $\mathrm{CCT}_{110} \%$, plasma lactate values were not different between groups. However, 5 min following the $\mathrm{CCT}_{110} \%$ (Fig. 6), a marked increase in plasma lactate was observed in $\mathrm{NaHCO}_{3}$ supplemented groups compared with $\mathrm{Pl}-\mathrm{Pl}$, significant only in the BAl-SB treatment.

The lack of any observed change in plasma lactate concentration between the BAl-Pl and Pl-Pl groups following both the RSA and $\mathrm{CCT}_{110}$ \% exercise protocols, suggests that enhanced intracellular buffering capacity did not promote increased glycolytic metabolism. Others have also reported no change in plasma lactate following HIE post chronic supplementation with $\beta$-alanine, although they did report performance benefits (Sweeney et al. 2010). Improvements in TTE in the current studies $\mathrm{CCT}_{110 \%}$ test may therefore indicate a potential mechanism of action other than enhanced intracellular buffering capacity in which $\beta$-alanine may provide ergogenic effects during continuous HIE.

In summary, there was no aggregating effect of the combined supplementation protocol on performance or metabolism, although the ingestion of $\beta$-alanine and $\mathrm{NaHCO}_{3}$ elevated both intra- and extracellular buffering capacity potential, by increasing concentrations of muscle carnosine and blood $\mathrm{HCO}_{3}{ }^{-}$, respectively. Alterations in buffering potential did coincide with performance enhancements in continuous fatiguing exercise, with TTE improved following $\beta$-alanine supplementation. No changes were found in performance parameters between the treatment combinations during maximal intermittent sprint exercise. No change in blood $\mathrm{pH}$ following $\mathrm{NaHCO}_{3}$ ingestion in the BAl-SB and Pl-SB groups demonstrates that an alkalotic state was not produced prior to exercise commencing, suggesting that the time between acute feeding and exercise implemented in this study may not be long enough to alter extracellular alkalinity, which in turn may have limited the potential for performance improvements. This may indicate that $\mathrm{pH}$ may not be a mechanism that influences fatigue in all HIE protocols. Further investigations into the potential mechanisms in which $\beta$-alanine provide ergogenic effect during continuous HIE performance, including the role of muscle carnosine as an antioxidant, are warranted as other intramuscular influences such as alterations in ROS (byproducts of antioxidant effects) may play an influential role in potentially enhancing exercise performance.

Author Contributions JD; Experimental design, conducted experimental trials, data collection and analysis, manuscript preparation. TG; Conducted experimental trials, data collection. RMW; data analysis. CGS; Experimental design, conducted experimental trials, data collection, manuscript preparation.

Acknowledgments Sincere thanks to Dr. Michael Kean (Chief MR Technologist) and staff of The Royal Children's Hospital (MR Centre Melbourne) for collection of MRS data. Dr. David Bishop and Dr. Alan Hayes for assistance with the experimental design implemented during this study and Dr. Matthew Cooke with assistance in manuscript preparation. Musashi Supplements ${ }^{\mathrm{TM}}$ Australia for generously providing $\beta$-alanine supplements. The additional assistance and support of the Exercise Metabolism Unit at Victoria University, Mr. Nikola Popovik and Ms. Dawn Bannon is also greatly appreciated.

Conflict of interest The authors declare that they have no conflict of interest.

Open Access This article is distributed under the terms of the Creative Commons Attribution License which permits any use, distribution, and reproduction in any medium, provided the original author(s) and the source are credited.

\section{References}

Baguet A, Reyngoudt H, Pottier A, Everaert I, Callens S, Achten E, Derave W (2009) Carnosine loading and washout in human skeletal muscles. J Appl Physiol 106:837-842 
Baguet A, Koppo K, Pottier A, Derave W (2010) $\beta$-alanine supplementation reduces acidosis but not oxygen uptake responses during high-intensity cycling exercise. Eur J Appl Physiol 108:495-503

Begum G, Cunliffe A, Leveritt M (2005) Physiological role of carnosine in contracting muscle. Int $\mathrm{J}$ Sport Nutr Exerc Metab 15:493-514

Bellinger PM, Howe ST, Shing CM, Fell JW (2012) Effect of combined $\beta$-alanine and sodium bicarbonate supplementation on cycling performance. Med Sci Sports Exerc 44:1545-1551

Bishop D, Edge J, Davis C, Goodman C (2004) Induced metabolic alkalosis affects muscle metabolism and repeated sprint ability. Med Sci Sports Exerc 36:807-813

Boldyrev AA, Severin SE (1990) The histidine-containing dipeptides, carnosine and anserine: distribution, properties, and biological significance. Adv Enzyme Regul 30:175-194

Chasovnikova L, Formazyuk V, Sergienko V, Boldyrev A, Severin S (1990) The anti-oxidative properties of carnosine and other drugs. Biochem Int 20:1097-1103

Coso JD, Hamouti N, Agudo-Jimenez R, Mora-Rodriguez R (2010) Restoration of blood $\mathrm{pH}$ between repeated bouts of high-intensity exercise: effects of various active-recovery protocols. Eur J Appl Physiol 108:523-532

Derave W, Özdemir MS, Harris RC, Pottier A, Reyngoudt H, Koppo K, Wise JA, Achten E (2007) $\beta$-alanine supplementation augments muscle carnosine content and attenuates fatigue during repeated isokinetic contraction bouts in trained sprinters. J Appl Physiol 103:1736-1743

Derave W, Everaert I, Beeckman S, Baguet A (2010) Muscle carnosine metabolism and $B$-alanine supplementation in relation to exercise and training. Sports Med 40:247-265

Fabiato A, Fabiato F (1978) Effects of $\mathrm{pH}$ on the myofilaments and the sarcoplasmic reticulum of skinned cells from cardiac and skeletal muscles. J Physiol 276:233-235

Fitzsimons M, Dawson B, Ward D, Wilkinson A (1993) Cycling and running tests of repeated sprint ability. Aust J Sci Med Sport 25:82-87

Gaitonos GC, Nevill ME, Brooks S, Williams C (1991) Repeated bouts of sprint running after induced alkalosis. J Sports Sci 9:355-369

Harris RC, Edwards RH, Hultman E, Nordesjo LO, Nylind B, Sahlin K (1976) The time course of phosphorylcreatine resynthesis during recovery of the quadriceps muscle in man. Pfugers Arch 367:137-142

Harris RC, Tallon MJ, Dunnett M, Boobis L, Coakley J, Kim HJ, Fallowfield JL, Hill CA, Sale C, Wise JA (2006) The absorption of orally supplied $\beta$-alanine and its effects on muscle carnosine synthesis in human vastus lateralis. Amino Acids 30:279-289

Hill CA, Harris RC, Kim HJ, Harris BD, Sale C, Boobis LH, Kim CK, Wise JA (2007) Influence of $\beta$-alanine supplementation on skeletal muscle carnosine concentrations and high intensity cycling capacity. Amino Acids 32:225-233

Hipkiss AR (2000) Carnosine and protein carbonyl groups: a possible relationship. Biochem (Moscow) 65:771-778

Hipkiss AR, Michaelis J, Syrris P (1995) Non-enzymatic glycosylation of the dipeptide L-carnosine, a potential anti-protein-crosslinking agent. FEBS Lett 371:81-85

Johnson P, Aldstaft J (1984) Effects of carnosine and anserine on muscle and non-muscle phosphorylases. J Comp Physiol B 78:331-333
Juel C, Klarskov C, Nielsen JJ, Krustrup P, Mohr M, Bangsbo J (2004) Effect of high-intensity intermittent training on lactate and $\mathrm{H}^{+}$release from human skeletal muscle. Am J Physiol Endocrinol Metab 286:245-251

Lindh AM, Peyrebrune MC, Ingham SA, Bailey DM, Folland JP (2008) Sodium bicarbonate improves swimming performance. Int J Sports Med 29:519-523

Matson LG, Vu Tran Z (1993) Effects of sodium bicarbonate ingestion on anaerobic performance: a meta-analytic review. Int $\mathrm{J}$ Sport Nutr 3:2-28

Matsuura R, Arimitsu T, Kimura T, Yunoki T, Yano T (2007) Effect of oral administration of sodium bicarbonate on surface EMG activity during repeated cycling sprints. Eur J Appl Physiol 101:409-417

McNaughton LR, Siegler J, Midgley A (2008) Ergogenic effects of sodium bicarbonate. Curr Sports Med Rep 7:230-236

Naressi A, Couturier C, Devos JM, Janssen M, Mangeat C, de Beer R, Graveron-Demilly D (2001) Java-based graphical user interface for the MRUI quantitation package. Magnet Reson Med $12: 141-152$

Price MJ, Simons C (2010) The effect of sodium bicarbonate ingestion on high-intensity intermittent running and subsequent performance. J Strength Cond 24:1834-1842

Price MJ, Moss P, Rance S (2003) Effects of sodium bicarbonate ingestion on prolonged intermittent exercise. Med Sci Sports Exerc 35:1303-1308

Robergs R, Hutchinson K, Hendee S, Madden S, Siegler J (2005) Influence of pre-exercise acidosis and alkalosis on the kinetics of acid-base recovery following intense exercise. Int J Sport Nutr 15:59-74

Sahlin K, Harris RC (2011) The creatine kinase reaction: a simple reaction with functional complexity. Amino Acids 40:1363-1367

Sale C, Saunders B, Harris RC (2010) Effects of $\beta$-alanine supplementation on muscle carnosine concentrations and exercise performance. Amino Acids 39:321-333

Sale C, Saunders B, Hudson S, Wise JA, Harris RC, Sunderland CD (2011) Effect of $\beta$-alanine plus sodium bicarbonate on highintensity cycling capacity. Med Sci Sports Exerc 43:1972-1978

Siegler JC, Keatley S, Midgley AW, Nevill AM, McNaughton LR (2007) Pre-exercise alkalosis and acid-base recovery. Int J Sports Med 29:545-551

Siegler JC, McNaughton LR, Midgley AW, Keatley S, Hillman A (2010) Metabolic alkalosis, recovery and sprint performance. Int J Sports Med 31:797-802

Sutton JR, Jone NL, Toews CJ (1981) Effects of pH on muscle glycolysis during exercise. Clin Sci (Lond) 61:331-338

Swank A, Robertson RJ (1989) Effect of induced alkalosis on perceptions of exertion during intermittent exercise. J Appl Physio 67:1862-1867

Sweeney KM, Wright GA, Brice AG, Doberstein ST (2010) The effect of $\beta$-alanine supplementation on power performance during repeated sprint activity. J Strength Cond 24:79-87

Van Thienen RV, Proeyen KV, Eynde BV, Puype J, Lefere T, Hespel $P$ (2009) $\beta$-alanine improves sprint performance in endurance cycling. Med Sci Sports Exerc 41:898-903

Vanhamme L, van den Boogaart A, Van Huffel S (1997) Improved method for accurate and efficient quantification of MRS data with use of prior knowledge. J Magn Reson 129:35-43 